\title{
Role of GnRH stimulation test in prediction of a neurogenic aetiology in girls with central precocious puberty
}

\author{
Saravana Bhavan, Hemchand Krishna Prasad, Anupama Sankaran, \\ Vijaykumar P Govindram, C V Ravisekar, K Nedunchelian Krishnamooorthy \\ Corresponding author: Dr. Hemchand Krishna Prasad, Address: 2, Mc Nichols Road, Chetpet, \\ Chennai Consultant, Department of pediatric Endocrinology, India; \\ Email : pediatricendocrinology.mehta@gmail.com
}

Distributed under Attribution-Non Commercial - Share Alike 4.0 International (CC BY-NC-SA 4.0)

\begin{abstract}
Objective: To ascertain the utility of gonadotrophin releasing hormone $(\mathrm{GnRH})$ test in diagnosis of neurogenic central precocious puberty (NCPP). Methods: We conducted a prospective observational study over a period of 4 years recruiting girls with early breast budding. Children underwent GnRH analogue stimulation test with injection luperide $20 \mathrm{mcg} / \mathrm{kg}$ in subcutaneous route followed by assessment of gonadotropins (LH and FSH) at 4 hours and estradiol at 24 hours. MRI performed in everyone with CPP. Cases divided into those with neurogenic CPP (NCPP) and idiopathic CPP (ICPP). Results: During the study period, 59 girls presented with early sexual maturation; we included 19 subjects onto our study (12 girls with idiopathic central precocious puberty and 7 girls with neurogenic precocious puberty). Stimulated LH was higher in subjects with NCPP compared to those with ICPP (9.8 \pm 3.0 versus 8.4 $\pm 3.6 \mathrm{mIU} / \mathrm{mL}, \mathrm{p}<0.05)$. Stimulated LH:FSH ratio was higher in those with NCPP versus ICPP $(0.5 \pm 0.3$ versus $0.34 \pm 0.3$, respectively). On multivariate regression analysis, stimulated LH:FSH ratio is the most likely determining factor for the occurrence of a neurogenic aetiology for central precocious puberty. ROC curve constructed to determine the optimal cut off to distinguish neurogenic and idiopathic causes of central precocious puberty. LH:FSH $>0.55$ is able to predict neurogenic precocious puberty with specificity of $82 \%$ and sensitivity of $66.7 \%$. Conclusion: Elevated LH levels and LH/FSH ratio in GnRHa stimulation test gives an important clue to a possible underlying neurological pathology. A cut-off of 0.55 predicts neurogenic precocity with a specificity of $82 \%$.
\end{abstract}

Keywords: Neurogenic, precocious puberty, gonadotrophin hormone, stimulation.

Precocious puberty is an important condition encountered by paediatricians and paediatric endocrinologists, in clinical practice ${ }^{1}$. Precocious puberty can be due to activation of hypothalamo pituitary gland signalling (central) or autonomous sex hormone production from gonads (peripheral) ${ }^{2}$. A methodical stepwise assessment is the key to arriving at the precise underlying aetiology ${ }^{3}$. The prevalence of central precocious puberty (CPP) is estimated to be 1 in 5000 to 10000 , with emerging evidence suggesting a rise in the prevalence ${ }^{4,5}$. A diagnosis of CPP has implications for a given child in terms of an underlying neurological disorder, final height prognosis and psychological well being of the given child. Children who have an underlying neurological condition like craniopharyngioma or hydrocephalus needs to be managed in a diametrically opposite way compared to children with central precocious puberty and normal magnetic resonance imaging (MRI) (Idiopathic). Thus, it is pivotal that children with idiopathic central precocious puberty (ICPP) and neurogenic central precocious puberty (NCPP) are distinguished properly by pediatricians and endocrinologists. Children with early menarche have difficulty in coping up with the physical changes, resulting in compromised academic performance. Clear cut guidelines have been established for the rational

Received: $27^{\text {th }}$ July 2020, Peer review completed: $14^{\text {th }}$ September 2020, Accepted: $1^{\text {st }}$ October 2020.

Bhavan S, Prasad HK, Sankaran A, Govindram VP, Ravisekar CV, Krishnamooorthy KN. Role of GnRH stimulation test in prediction of a neurogenic aetiology in girls with central precocious puberty. The New Indian Journal of OBGYN. 2021; 8(1): 57-61. 
therapy of children with precocious puberty ${ }^{6}$. Diagnosis of CPP needs documentation of pubertal hypothalamo pituitary gonadal (HPG) axis. The gonadotrophin analogue stimulation test is a useful test to document the pubertal HPG axis in children with precocious puberty ${ }^{7}$. The utility of the GnRH analogue stimulation test in distinguishing ICPP and NCPP is inconclusive ${ }^{8,9}$. Hence, we decided to perform this study to assess the utility of GnRH analogue (GnRHa) testing distinguish NCPP and ICPP.

\section{Methods}

We conducted a prospective observational study over a period of 4 years. All girls presenting to the endocrine clinic with concerns of early breast budding were recruited onto the study. They were assessed clinically as per standard protocols and evaluated. We excluded subjects with peripheral precocious puberty and features of heterosexual precocious puberty. All girls underwent Tanner's stage assessment, anthropometric measurement as per standard protocols. A meticulous history for neurological symptoms and clinical neurological examination was performed in all children. Bone age assessment was performed by the Tanner Whitehouse-3 method and ultrasound pelvis was performed by a single paediatric radiologist to look at size of pelvic organs. A basal thyroid profile was performed in all subjects. Basal leutinizing hormone ( $\mathrm{LH})$, follicle stimulating hormone (FSH) and estradiol were assayed. Children underwent $\mathrm{GnRH}$ analogue stimulation test with injection luperide $20 \mathrm{mcg} / \mathrm{kg}$ in subcutaneous route (procured from sun pharma). This was followed by assessment of gonadotrophins (LH and FSH) at 4 hours and estradiol at 24 hours. Girls with peak LH levels $>5 \mathrm{mIU} / \mathrm{ml}$ and stimulated estradiol $>50 \mathrm{pg} / \mathrm{mL}$ were considered as central precocious puberty (cases) and girls with peak LH levels $<5 \mathrm{mIU} / \mathrm{ml}$, stimulated estradiol $<50 \mathrm{pg} / \mathrm{mL}$ and/or no progression in Tanner staging, anthropometry and pelvic organs as simple thelarche. Subjects with simple thelarche were followed up in the endocrine clinic for pubertal progression and growth. Subjects diagnosed as CPP underwent an MRI brain. All subjects underwent magnetic resonance imaging at a single centre. $1.5 \mathrm{~mm}$ contiguous sagittal and coronal $\mathrm{T} 1$ and $\mathrm{T} 2$ weighted MR images using 1.5 tesla (Siemens Magnetom Era) was used to perform imaging as per standard pituitary protocol (10). All MRI images were reported by a single radiologist, who was blinded to details of the study sample.

They were initiated on GnRH analogue therapy with injection lupride $(0.25 \mathrm{mg} / \mathrm{kg})$ deep intramuscularly monthly once, if they were found to be progressive.
Data was entered in microsoft excel sheet. Statistical analysis was done with SPSS software version 22.0. Anthropometric parameters SD score was calculated based on WHO standards and IAP 2015 references ${ }^{11,12}$. Pelvic organ dimensions converted into Z-scores ${ }^{13}$. Chi-square test and student t-test to compare clinical and biochemical and radiological parameters in cases and controls, as appropriate, $\mathrm{p}<0.05$ was considered as significant. Receiver operating curve (ROC) was utilized to compute the optimal gonadotrophin level to distinguish NCPP and ICPP.

\section{Results}

During the study period, 59 girls presented with early sexual maturation; after excluding subjects with heterosexual precocious puberty $(\mathrm{n}=10)$, girls with simple thelarche $(n=23)$ and peripheral precocious puberty $(n=7)$, we included 19 subjects onto our study (12 girls with idiopathic central precocious puberty and 7 girls with neurogenic precocious puberty). The underlying cause for NCPP was craniopharyngioma, pituitary adenoma, arachnoid cyst, tuberculoma and hypothalamic hamartoma in 2,2,1,1 and 1 case each respectively. Seizures and developmental delay was present in one case each.

Table 1: Comparison of clinical, demographic, anthropometric and radiological parameters in subjects with ICPP and NCPP

\begin{tabular}{lll}
\hline Parameters & ICPP (n=12) & NCPP (n=7) \\
\hline Chronological age (in years) & $5.4 \pm 1.3$ & $5.8 \pm 1.7$ \\
Height (in cm) & $115.1 \pm 22.0$ & $114.8 \pm 26.0$ \\
BMI (in kg/m ${ }^{2}$ ) & $17.8 \pm 1.9$ & $16.9 \pm 1.6$ \\
Height SD score & $1.4 \pm 0.3$ & $1.8 \pm 0.34$ \\
BMI SD score & $0.7 \pm 0.2$ & $0.6 \pm 0.2$ \\
Tanner stage & & \\
B2 & $4(33.33 \%)$ & $1(14.2 \%)$ \\
B3 & $8(66.66 \%)$ & $6(85.71 \%)$ \\
P2 & $4(33.33 \%)$ & $1(14.2 \%)$ \\
P3 & $8(66.66 \%)$ & $6(85.71 \%)$ \\
Bone age (in years) & $7.6 \pm 0.9$ & $6.5 \pm 1.0$ \\
Chronological age: bone age ratio & $1.3 \pm 0.3$ & $1.1 \pm 0.2$ \\
Uterine length (in cm) & $4.5 \pm 0.4$ & $4.1 \pm 0.3$ \\
Ovarian Volume (in cm ${ }^{3}$ ) & $1.7 \pm 0.8$ & $1.9 \pm 0.4$ \\
Uterine length Z-score & $1.2 \pm 0.3$ & $1.3 \pm 0.2$ \\
Ovarian volume Z-scores & $2.2 \pm 0.4$ & $1.4 \pm 0.3$ \\
\hline \multicolumn{2}{l}{ The baseline clinical }
\end{tabular}

The baseline clinical, demographic, anthropometric and radiological parameters are presented in table-1. The chronological age, bone age, bone age: chronological age ratio, anthropometric parameters (absolute value and $\mathrm{Z}$ score), uterine length, ovarian volume and their corresponding Z-scores are similar in subjects with NCPP and ICPP.

The biochemical parameters of subjects with NCPP and ICPP are compared in table - 2. It was observed that basal gonadotrophins, stimulated FSH and estradiol are comparable in the two study groups. However, it was observed that stimulated LH was higher in subjects with 
NCPP compared to those with ICPP $(9.8 \pm 3.0$ versus $8.4 \pm 3.6$ $\mathrm{mIU} / \mathrm{mL}, \mathrm{p}<0.05)$. It was also noted that stimulated LH:FSH ratio was higher in those with NCPP versus ICPP $(0.5 \pm 0.3$ versus $0.34 \pm 0.3$, respectively). These are depicted in figure 1 and 2.

Table 2: Comparison of biochemical parameters in subjects with ICPP and NCPP

\begin{tabular}{lll}
\hline Parameters & ICPP $(\mathbf{n}=\mathbf{1 2})$ & NCPP $(\mathbf{n}=7)$ \\
\hline Basal LH & $0.6 \pm 1.1$ & $0.8 \pm 1.2$ \\
Basal FSH & $3.7 \pm 1.8$ & $3.1 \pm 0.4$ \\
Stimulated LH* & $8.4 \pm 3.6$ & $9.8 \pm 3.0$ \\
Stimulated FSH & $21.7 \pm 2.3$ & $19.4 \pm 9.1$ \\
LH:FSH ratio* & $0.34 \pm 0.3$ & $0.5 \pm 0.3$ \\
E2 & $43.0 \pm 16$ & $39.6 \pm 13.5$ \\
\hline
\end{tabular}

On univariate analysis, it was observed that stimulated LH and stimulated LH: FSH ratio is determinants of occurrence of neurogenic precocity. On multivariate regression analysis, it was observed that stimulated LH:FSH ratio is the most likely determining factor for the occurrence of a neurogenic aetiology for central precocious puberty.

\begin{tabular}{|c|c|c|c|c|}
\hline \multirow[b]{2}{*}{ Parameters } & & \multicolumn{2}{|c|}{$\begin{array}{l}\text { Unstandardized } \\
\text { Coefficients }\end{array}$} & \multirow{2}{*}{$\begin{array}{l}\text { Standardized } \\
\text { Coefficients } \\
\text { Beta } \\
\end{array}$} \\
\hline & & B & Std. Error & \\
\hline \multicolumn{2}{|l|}{ Height Z-score } & -0.176 & 0.058 & -1.151 \\
\hline \multicolumn{2}{|c|}{ Bone age: Chronological age ratio } & 0.282 & 0.612 & 0.117 \\
\hline \multicolumn{2}{|l|}{ Basal LH } & -0.606 & 0.165 & -1.559 \\
\hline \multicolumn{2}{|l|}{ Basal FSH } & 0.335 & 0.143 & 1.492 \\
\hline \multicolumn{2}{|l|}{ Stimulated LH } & -0.442 & 0.102 & -5.811 \\
\hline \multicolumn{2}{|l|}{ Stimulated FSH } & 0.061 & 0.024 & 0.922 \\
\hline \multicolumn{2}{|l|}{ LH: FSH ratio } & 10.833 & 2.288 & 5.750 \\
\hline \multicolumn{2}{|l|}{ Chronological age } & 0.008 & 0.058 & 0.026 \\
\hline \multicolumn{5}{|c|}{$\begin{array}{l}\text { A receiver operating curve (ROC) curve was constructed } \\
\text { to determine the optimal cut off to distinguish neurogenic }\end{array}$} \\
\hline \multicolumn{5}{|l|}{12} \\
\hline \multicolumn{5}{|l|}{10} \\
\hline \multicolumn{5}{|l|}{8} \\
\hline \multicolumn{5}{|l|}{6} \\
\hline \multicolumn{5}{|l|}{4} \\
\hline \multicolumn{5}{|l|}{2} \\
\hline \multicolumn{5}{|c|}{ ICPP } \\
\hline $\begin{array}{c}\text {-S Stimulated LH level } \\
(\mathrm{mIU} / \mathrm{mL})\end{array}$ & 8.4 & & 9.8 & \\
\hline
\end{tabular}

Legend: ICPP - Idiopathic Central Precocious Puberty, NCPP - Neurogenic Central Precocious Puberty

Figure 1: Comparison of Stimulated LH level in subjects with ICPP and NCPP

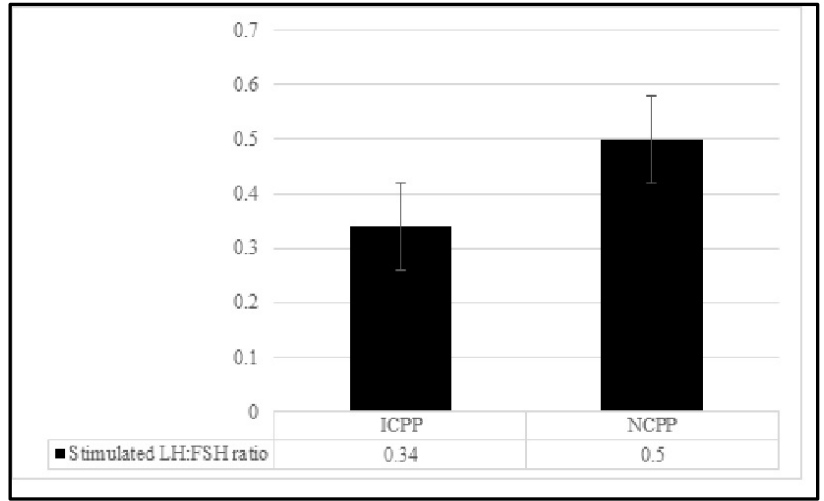

Legend: ICPP - Idiopathic Central Precocious Puberty, NCPP - Neurogenic Central Precocious Puberty

Figure 2: Comparison of stimulated LH: FSH ratio in subjects with ICPP and NCPP

and idiopathic causes of central precocious puberty. LH:FSH $>0.55$ is able to predict neurogenic precocious puberty with specificity of $82 \%$ and sensitivity of $66.7 \%$ (figure 3 ). In our study 4 girls were in age group 6-8 yrs with neurogenic CPP all had stimulated LH:FSH $>0.55$. Subjects with NCPP were managed as per the underlying aetiology. Out of the nineteen subjects 15 were initiated on long term GnRH analogue therapy with Inj luperide $0.25 \mathrm{mg} / \mathrm{kg}$ as a monthly depot.

\section{Discussion}

In our study, we have dogmatically demonstrated that GnRH analogue stimulation test is indeed a useful tool in the hands of the

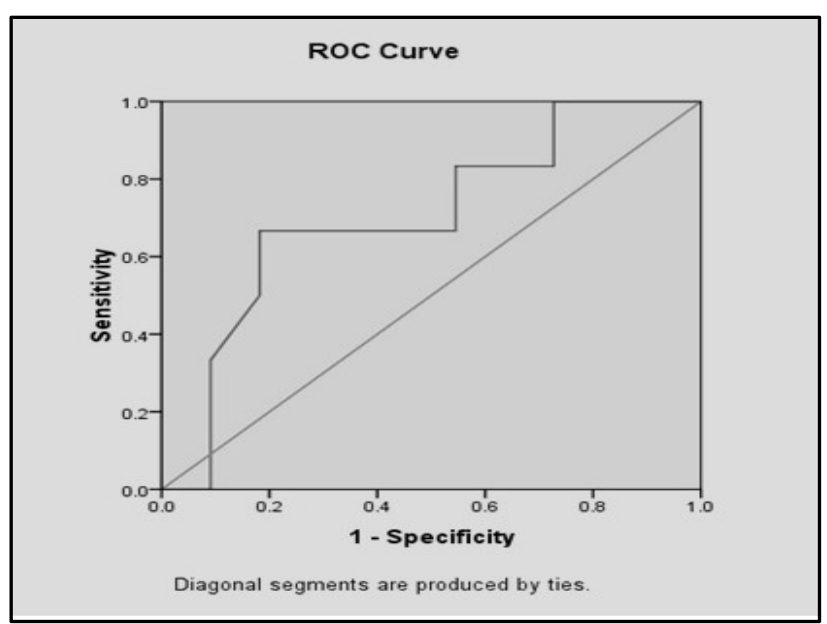

Figure 3: ROC curve determine the optimal LH: FSH ratio cut off to identify neurogenic central precocious puberty 
The New Indian Journal of OBGYN. 2021 (July-December);8(1)

paediatrician to recognize subjects with an underlying neurological aetiology. We observed that, in our subjects 7 out of $19(36.9 \%)$ of subjects had an underlying neurological cause of CPP. Out of these seven, three were $<6$ years and four were more than 6 years. This is in line with another Indian study which has reported that $43.8 \%$ of girls with neurogenic CPP had age of onset 6-8 years ${ }^{14}$. Thus, it is mandatory pediatricians treating CPP should perform an MRI in girls with CPP aged 6-8 years in the slightest index of suspicion.

We observed that, stimulated LH was higher in subjects with NCPP compared to those with ICPP $(9.8 \pm 3.0$ versus $8.4 \pm 3.6 \mathrm{mIU} / \mathrm{mL}, \mathrm{p}<0.05)$. This is contrary to the observation of previous reports that early age of onset is the only predictor of neurogenic precocity ${ }^{8}$. A previous study on 67 girls have revealed that none of the parameters assessed in the GnRH stimulation test are likely to determine a neurogenic cause ${ }^{15}$. We hypothesise that subtle differences in neuroendocrine regulation in those with NCPP result in more rapid pubertal maturation resulting in higher LH levels than ICPP group ${ }^{16}$.

We also noted that stimulated LH:FSH ratio was higher in those with NCPP versus ICPP $(0.5 \pm 0.3$ versus $0.34 \pm 0.3$, respectively). Bajpai $\mathrm{A}$ et al ${ }^{14}$ have reported that elevated stimulated $\mathrm{LH}$ and $\mathrm{LH} / \mathrm{FSH}$ ratio are strong pointers to a neurogenic cause. We noted that LH:FSH $>0.55$ is able to predict neurogenic precocious puberty with specificity of $82 \%$ and sensitivity of $66.7 \%$. The mechanism of this failure in the inactivation of GnRH is unknown. Data suggest that in neurological lesions, an unknown factor, probably secreted by the tumours, advances the tempo of GnRH maturation ${ }^{17}$. Therefore, the pubertal hormones are impacted by the aetiology of organic central precocious puberty related to location and type of lesion ${ }^{17}$.

\section{Conclusion}

We conclude that elevated $\mathrm{LH}$ levels and $\mathrm{LH} / \mathrm{FSH}$ ratio in GnRHa stimulation test gives an important clue to a possible underlying neurological pathology. A cut - off of 0.55 predicts neurogenic precocity with a specificity of $82 \%$.

Acknowledgement - We would like to thank all the children and their families who participated in the study

\section{Conflict of interest: None. Disclaimer: Nil.}

\section{References}

1. Rosenfield RL, Lipton RB, Drum ML. Thelarche, pubarche, and menarche attainment in children with normal and elevated body mass index. Pediatrics. 2009;123(1):84-8.

2. Mogensen SS, Aksglaede L, Mouritsen A, Sorensen K, Main KM, Gideon P, et al. Diagnostic work-up of 449 consecutive girls who were referred to be evaluated for precocious puberty. J Clin Endocrinol Metab. 2011; 96(5):1393-401

3. Fuqua JS. Treatment and outcomes of precocious puberty: an update. J Clin Endocrinol Metab. 2013; 98(6): 2198-207.

4. Partsch CJ, Sippell WG. Pathogenesis and epidemiology of precocious puberty. Effects of exogenous oestrogens. Hum Reprod Update. 2001;7:292-302.

5. Teilmann G, Pedersen CB, Jensen TK, Skakkebaek NE, Juul A. Prevalence and incidence of precocious pubertal development in Denmark: an epidemiologic study based on national registries. Pediatrics. 2005;116:1323-8.

6. Carel JC, Eugster EA, Rogol A, Ghizzoni L, Palmert MR, ESPE-LWPES GnRH Analogs Co analogs nsensus Conference Group, et al. Consensus statement on the use of gonadotropin - releasing hormone in children. Pediatrics. 2009;123:e752-62.

7. Prasad HK, Khadilkar VV, Jahagirdar R, Khadilkar AV, Lalwani SK. Evaluation of GnRH analogue testing in diagnosis and management of children with pubertal disorders. Indian J Endocrinol Metab. 2012;16:400-5

8. Choi JH, Shin YL, Yoo HW. Predictive factors for organic central precocious puberty and utility of simplified gonadotropin - releasing hormone tests. Pediatr. Int. 2007; 49: 806-10.

9. Bizzarri C, Spadoni GL, Bottaro G, Montanari G, Giannone G, Cappa M, Cianfarani S. The response to gonadotropin releasing hormone $(\mathrm{GnRH})$ stimulation test does not predict the progression to true precocious puberty in girls with onset of premature thelarche in the first three years of life. J Clin Endocrinol Metab. 2014; 99:433-9

10. Tsai SL, Laffan E. Congenital growth hormone deficiency: a review focus on neuroimaging. Eur Endocrinol. 2013; 9:136-40

11. de Onis M, Onyango AW, Borghi E, Siyam A, Nishida C, Siekmann J, et al. Development of a WHO growth reference for school-aged children and adolescents. Bull World Health Organ. 2007; 85: 660-7.

12. Khadilkar V, Yadav S, KK Agrawal, Tamboli S, Banerjee M, Cherian A et al. Revised IAP Growth Charts for Height, Weight and Body Mass Index for 5- 
The New Indian Journal of OBGYN. 2021 (July-December);8(1)

to 18-year-old Indian Children. Indian Pediat. 2015 Jan; 52(1); 47-55.

13. Khadilkar VV, Khadilkar AV, Kinare AS, Tapasvi HS, Deshpande SS, Maskati GB. Ovarian and Uterine ultrasonography in healthy girls between birth and 18 years. Indian Pediatrics. 2006 ;43:625-30

14. Bajpai A, Sharma J, Kabra M, Kumar Gupta A, Menon PS. Precocious puberty: Clinical and endocrine profile and factors indicating neurogenic precocity in Indian children. J Pediatr Endocrinol Metab. 2002;15:1173-81

15. Ng SM, Kumar Y, Cody D, Smith C, Didi M. The gonadotrophins response to $\mathrm{GnRH}$ test is not a predictor of neurological lesion in girls with central precocious puberty. Journal of Pediatric Endocrinology and Metabolism. 2005;18(9): 849-52.

16. Uriarte MM, Klein KO, Barnes KM, Pescovitz $\mathrm{OH}$, Loriaux DL, et al. Gonadotrophin and prolactin secretory dynamics in girls with normal puberty, idiopathic precocious puberty and precocious puberty due to hypothalamic hamartoma. Clin Endocrinol (Oxf) . 1998 Sep; 49(3):363-8
17. Rivarola MA, Belgorosky A, Mendilaharzu H, Vidal G. Precocious puberty in children with tumours of the suprasellar and pineal areas: organic central precocious puberty. Acta Paediatr. 2001; 90(7):751-56.

Saravana Bhavan ${ }^{1}$, Hemchand Krishna Prasad ${ }^{2}$,
Anupama Sankaran ${ }^{3}$, Vijaykumar P Govindram ${ }^{4}$, C V
Ravisekar ${ }^{5}$, K Nedunchelian Krishnamooorthy ${ }^{6}$
${ }^{1}$ DNB Pediatric Trainee, Mehta Multispeciality
Hospitals India Pvt Ltd, Chennai, India; ${ }^{2}$ Consultant,
Department of Pediatric Endocrinology, Mehta
Multispeciality Hospitals India Pvt Ltd, Chennai, India; ${ }^{3}$
Pediatrician, Mehta Multispeciality Hospitals India Pvt
Ltd, Chennai, India; ${ }^{4}$ Consultant, Department of
Biochemistry, Mehta Multispeciality Hospitals India
Pvt Ltd, Chennai, India; ${ }^{5}$ Consultant Pediatrician,
Mehta Multispeciality Hospitals India Pvt Ltd, Chennai,
India; ${ }^{6}$ Consultant Pediatrician, Mehta Multispeciality
Hospitals India Pvt Ltd, Chennai, India.

